Article

\title{
Microwave Accelerated Aza-Claisen Rearrangement
}

\section{Eva Gajdošíková, Miroslava Martinková, Jozef Gonda* and Patrik Čonka}

Institute of Chemical Sciences, Department of Organic Chemistry, P. J. Šafárik University, Moyzesova 11, SK-040 01 Košice, Slovak Republic; E-mails: eva.gajdosikova@gmail.com (E. G.), miroslava.martinkova@upjs.sk (M. M.),patrik.conka@gmail.com (P. C.)

* Author to whom correspondence should be addressed; E-mail: jozef.gonda@upjs.sk; Tel.: +421-55 6228332; Fax: +421-55 6222421.

Received: 7 October 2008; in revised form: 28 October 2008 / Accepted: 4 November 2008 / Published: 14 November 2008

\begin{abstract}
A study of microwave-induced and standard thermal Overman rearrangement of selected allylic trichloroacetimidates 1a-1f, 6-8 to the corresponding acetamides 2a-2f, 9$\mathbf{1 1}$ is reported. The microwave-assisted rearrangement of trifluoroacetimidate $\mathbf{1 3}$ is also described. Using this methodology, an efficient access to versatile allylic trihaloacetamides building synthons was established.
\end{abstract}

Keywords: Overman rearrangement; Imidate; Microwave irradiation.

\section{Introduction}

The [3,3]-sigmatropic rearrangement of allylic trihaloacetimidates into allylic trihaloacetamides is a useful methodology for the synthesis of nitrogen containing compounds such as amino acids [1-3], modified nucleosides [4,5] or other complex biologically interesting products [6-9]. This transformation is very often involved as the key step in the synthetic approaches and can be accomplished either at elevated temperatures or catalyzed by metal salts such as $\mathrm{Hg}\left(\mathrm{OCOCF}_{3}\right)_{2}$ [10,11], $\mathrm{PdCl}_{2}$ complexes [12,13] and new $\mathrm{Pt}(\mathrm{II}), \mathrm{Pt}(\mathrm{IV}), \mathrm{Au}(\mathrm{I})$ and $\mathrm{Au}(\mathrm{III})$ catalysts [14] under very mild reaction conditions. 
A significant acceleration of aza-Claisen rearrangements was observed using microwave irradiation [15]. This fact eliminated problems with previously required high temperatures and extended reaction times, and also reduced decomposition of the starting materials and products.

\section{Results and Discussion}

In this communication, we wish to report on microwave-assisted thermal Overman rearrangement of some selected allylic trihaloacetimidates 1a-f, 6-8, 13 that are derived either from simple allylic alcohols, amino acids or the modified sugars, respectively, and thus illustrate the potential of microwave irradiation to accelerate this reaction.

Scheme 1. Microwave accelerated Overman rearrangement of simple aliphatic imidates.<smiles>[R]C([R])=CC([R3])OC(=N)C(Cl)(Cl)Cl</smiles>

1

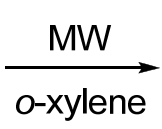

o-xylene<smiles>[R3]C=CC([R2])NC(=O)[O-]</smiles>

2

Table 1. Microwave accelerated Overman rearrangement of simple aliphatic imidates.

\begin{tabular}{|c|c|c|c|c|}
\hline Entry & $\mathbf{R}$ & Conditions & Time & $\begin{array}{c}\text { Yield }^{\mathrm{a}}(\%) \\
\text { 2a-f } \\
\end{array}$ \\
\hline \multirow{3}{*}{1} & \multirow{3}{*}{$\mathrm{R}^{1}=\mathrm{H}, \mathrm{R}^{2}=\mathrm{H}, \mathrm{R}^{3}=\mathrm{H}$ (a) } & $\Delta, 140^{\circ} \mathrm{C}, o$-xylene $[10,15]$ & $12 \mathrm{~h}$ & 50 \\
\hline & & $\mathrm{MW}, 140{ }^{\circ} \mathrm{C}, o$-xylene, $\mathrm{K}_{2} \mathrm{CO}_{3}$ & $10 \mathrm{~h}$ & 65 \\
\hline & & $\mathrm{MW}, 210{ }^{\circ} \mathrm{C}, o$-xylene, $\mathrm{K}_{2} \mathrm{CO}_{3}{ }^{\mathrm{b}}$ & $5 \mathrm{~min}$ & 70 \\
\hline \multirow{4}{*}{2} & \multirow{4}{*}{$\mathrm{R}^{1}=\mathrm{H}, \mathrm{R}^{2}=\mathrm{H}, \mathrm{R}^{3}=\mathrm{Me}(\mathbf{b})$} & $\Delta, 110^{\circ} \mathrm{C}$, toluene $[18]$ & $2 \mathrm{~h}$ & 53 \\
\hline & & $\Delta, 140^{\circ} \mathrm{C}, o$-xylene, $\mathrm{K}_{2} \mathrm{CO}_{3}$ & $2 \mathrm{~h}$ & 60 \\
\hline & & $\mathrm{MW}, 140{ }^{\circ} \mathrm{C}, o$-xylene, $\mathrm{K}_{2} \mathrm{CO}_{3}[16]$ & $15 \mathrm{~min}$ & 93 \\
\hline & & $\mathrm{MW}, 180{ }^{\circ} \mathrm{C}, o$-xylene, $\mathrm{K}_{2} \mathrm{CO}_{3}{ }^{\mathrm{b}}[16]$ & $8 \mathrm{~min}$ & 89 \\
\hline \multirow{4}{*}{3} & \multirow{4}{*}{$\mathrm{R}^{1}=\mathrm{H}, \mathrm{R}^{2}=\mathrm{H}, \mathrm{R}^{3}=\mathrm{Bu}(\mathbf{c})$} & $\Delta, 140{ }^{\circ} \mathrm{C}, o$-xylene $[10]$ & $2.5 \mathrm{~h}$ & 74 \\
\hline & & $\Delta, 140{ }^{\circ} \mathrm{C}, o$-xylene, $\mathrm{K}_{2} \mathrm{CO}_{3}$ & $2.5 \mathrm{~h}$ & 75 \\
\hline & & $\mathrm{MW}, 140{ }^{\circ} \mathrm{C}, o$-xylene, $\mathrm{K}_{2} \mathrm{CO}_{3}[16]$ & $5 \mathrm{~min}$ & 97 \\
\hline & & $\mathrm{MW}, 180^{\circ} \mathrm{C}, o$-xylene, $\mathrm{K}_{2} \mathrm{CO}_{3}{ }^{\mathrm{b}}[16]$ & $1 \mathrm{~min}$ & 94 \\
\hline \multirow{2}{*}{4} & \multirow{2}{*}{$\mathrm{R}^{1}=\mathrm{H}, \mathrm{R}^{2}=\mathrm{H}, \mathrm{R}^{3}=$ Pent (d) } & $\Delta, 140{ }^{\circ} \mathrm{C}, o$-xylene, $\mathrm{K}_{2} \mathrm{CO}_{3}$ & $1.5 \mathrm{~h}$ & 97 \\
\hline & & $\mathrm{MW}, 140{ }^{\circ} \mathrm{C}, o$-xylene, $\mathrm{K}_{2} \mathrm{CO}_{3}$ & $5 \mathrm{~min}$ & 92 \\
\hline \multirow{4}{*}{5} & \multirow{4}{*}{$\mathrm{R}^{1}=\mathrm{Me}, \mathrm{R}^{2}=\mathrm{Me}, \mathrm{R}^{3}=\mathrm{H}$ (e) } & $\Delta, 140{ }^{\circ} \mathrm{C}, o$-xylene $[11]$ & $3.5 \mathrm{~h}$ & 48 \\
\hline & & $\Delta, 140{ }^{\circ} \mathrm{C}, o$-xylene, $\mathrm{K}_{2} \mathrm{CO}_{3}$ & $3.5 \mathrm{~h}$ & 80 \\
\hline & & $\mathrm{MW}, 140{ }^{\circ} \mathrm{C}, o$-xylene, $\mathrm{K}_{2} \mathrm{CO}_{3}[16]$ & $20 \mathrm{~min}$ & 85 \\
\hline & & $\mathrm{MW}, 180^{\circ} \mathrm{C}, o$-xylene, $\mathrm{K}_{2} \mathrm{CO}_{3}{ }^{\mathrm{b}}[16]$ & $14 \mathrm{~min}$ & 84 \\
\hline \multirow{2}{*}{6} & \multirow{2}{*}{$\mathrm{R}^{1}=\mathrm{Me}, \mathrm{R}^{2}=\mathrm{H}, \mathrm{R}^{3}=\mathrm{Me}(\mathbf{f})$} & $\Delta, 140{ }^{\circ} \mathrm{C}, o$-xylene, $\mathrm{K}_{2} \mathrm{CO}_{3}$ & $45 \mathrm{~min}$ & 70 \\
\hline & & $\mathrm{MW}, 140{ }^{\circ} \mathrm{C}, o$-xylene, $\mathrm{K}_{2} \mathrm{CO}_{3}$ & $45 \mathrm{~min}$ & 75 \\
\hline
\end{tabular}

${ }^{\mathrm{a}}$ Isolated yield. ${ }^{\mathrm{b}} \mathrm{MW}$ experiments were performed in the presence of a heating bar, Weflon, Milestone. 
Thermally driven [3,3]-sigmatropic rearrangements (Scheme 1) were carried out according to the procedure described by Overman [10]. In the microwave-assisted thermal aza-Claisen rearrangement, the imidate was dissolved in $o$-xylene, powdered anhydrous $\mathrm{K}_{2} \mathrm{CO}_{3}$ [17] $(2 \mathrm{mg} / \mathrm{mL})$ was added, and the solution was heated under sealed vessel conditions. The scope of this method was investigated and all synthesized imidates (only imidates $\mathbf{1 e}$ and $\mathbf{1 f}$ were not characterized and used immediately to avoid problems connected with their instability) in Table 1 were converted to the corresponding trichloroacetamides $\mathbf{2 a - 2 f}$ in considerably shorter reaction times, compared to the conventional thermal rearrangement. We have observed that the use of microwave irradiation lead to a substantial reduction of the reaction times (from hours to minutes, Table 1, Entry 1-5). On the other hand, the conversion of $\mathbf{1 f}$ to compound $\mathbf{2 f}$ was achieved at the same reaction time in the both cases (the microwave-assisted and standard thermal conditions, Table 1, Entry 6).

Scheme 2. Microwave accelerated Overman rearrangement of the chiral imidates.

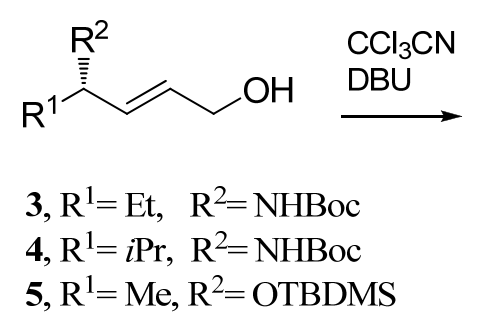

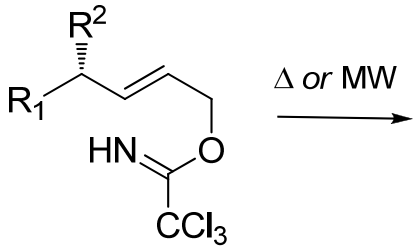

$6,7,8$

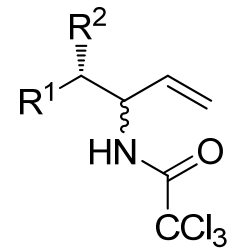

9, 10, 11

Table 2. Microwave accelerated Overman rearrangement of the chiral imidates 6, 7, 8 and 13.

\begin{tabular}{|c|c|c|c|c|}
\hline Entry & $\begin{array}{l}\text { Starting } \\
\text { material } \\
\end{array}$ & Conditions & Time & Yield $^{\mathrm{a}}(\%)$ \\
\hline \multirow{3}{*}{1} & \multirow{3}{*}{6} & $\Delta, 140{ }^{\circ} \mathrm{C}, o$-xylene, $d e=10 \%[12]$ & $24 \mathrm{~h}$ & 75 \\
\hline & & $\mathrm{MW}, 140{ }^{\circ} \mathrm{C}, o$-xylene, $\mathrm{K}_{2} \mathrm{CO}_{3} d e=14 \%[16]$ & $2 \mathrm{~h}$ & 80 \\
\hline & & $\mathrm{MW}, 200{ }^{\circ} \mathrm{C}, o$-xylene, $\mathrm{K}_{2} \mathrm{CO}_{3} d e=12 \%$ [16] & $5 \mathrm{~min}$ & 80 \\
\hline \multirow{3}{*}{2} & \multirow{3}{*}{7} & $\Delta, 140{ }^{\circ} \mathrm{C}, o$-xylene, $d e=10 \%[12]$ & $24 \mathrm{~h}$ & 69 \\
\hline & & $\mathrm{MW}, 140{ }^{\circ} \mathrm{C}, o$-xylene, $\mathrm{K}_{2} \mathrm{CO}_{3} d e=12 \%$ [16] & $2 \mathrm{~h}$ & 71 \\
\hline & & $\mathrm{MW}, 200{ }^{\circ} \mathrm{C}, o$-xylene, $\mathrm{K}_{2} \mathrm{CO}_{3} d e=13 \%$ [16] & $5 \mathrm{~min}$ & 68 \\
\hline \multirow{2}{*}{3} & \multirow{2}{*}{8} & $\Delta, 140{ }^{\circ} \mathrm{C}, o$-xylene, $d e=2 \%$ & $42 \mathrm{~h}$ & 80 \\
\hline & & $\mathrm{MW}, 160{ }^{\circ} \mathrm{C}, o$-xylene, $\mathrm{K}_{2} \mathrm{CO}_{3} d e=2 \%$ & $1 \mathrm{~h}$ & 86 \\
\hline \multirow{3}{*}{4} & \multirow{3}{*}{13} & $\Delta, 180{ }^{\circ} \mathrm{C}, o$-xylene, $\mathrm{K}_{2} \mathrm{CO}_{3}, d e \approx 20 \%$ & $12 \mathrm{~h}$ & 31 \\
\hline & & $\mathrm{MW}, 180^{\circ} \mathrm{C}, o$-xylene, $\mathrm{K}_{2} \mathrm{CO}_{3}$, de $\approx 19 \%$ & $30 \mathrm{~min}$ & 68 \\
\hline & & $\mathrm{MW}, 180^{\circ} \mathrm{C}, o$-xylene, $d e \approx 18 \%$ & $1 \mathrm{~h}$ & 15 \\
\hline
\end{tabular}

In earlier studies was found that Pd(II)-catalyzed Overman rearrangement of trichloroacetimidates 6, 7 derived from primary allylic alcohols with an adjacent centre of chirality proceeded with an excellent diastereoselectivity ( $d e \geq 98 \%$ ) [12]. In the next phase of our work we decided to study whether the described microwave-assisted Overman rearrangement could lead to a certain degree of 
diastereoselection. The conversion of known allylic alcohols $[12,14]$ into trichloroacetimidates 6-8 was achieved using trichloroacetonitrile and DBU as a base in dichloromethane (Scheme 2). The results of the thermal and microwave induced Overman rearrangements of imidates 6-8 are summarized in Table 2. We have found that microwave irradiation of 6- $\mathbf{8}$ led to the rearranged products $\mathbf{9 ,} \mathbf{1 0}$ [12] and 11 [14] (as the mixtures of diastereoisomers) with substantial shortening of the reaction times (from $24 \mathrm{~h}$ to $5 \mathrm{~min}$ ) with good yields (Table 2), however, it has shown that in these cases microwave-induced rearrangement had practically no influence on the diastereoselectivity of aza-Claisen rearrangement (Table 2).

Scheme 3. Microwave accelerated Overman rearrangement of the sugar trifluoroacetimidate 13.

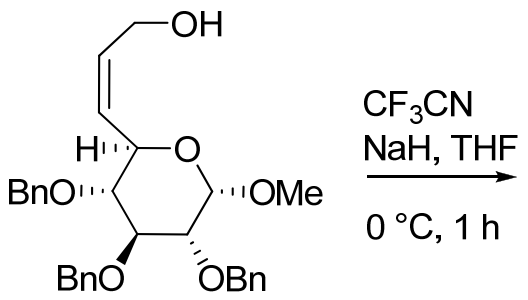

12<smiles>CO[C@H]1O[C@H](OCc2ccccc2)[C@@H](Oc2ccccc2)[C@@H](O)[C@@H]1OCC(F)(F)F</smiles>

13

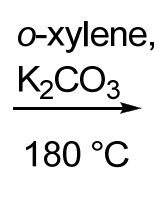

$\mathrm{BnO}$

$14 a, b$

Finally, we have investigated Overman rearrangement of the sugar allylic trifluoroacetimidate $\mathbf{1 3}$ under microwave irradiation. Trifluoroacetimidate 13 was prepared from the corresponding allylic alcohol 12 derived from D-glucose [19] by reaction with $\mathrm{CF}_{3} \mathrm{CN}$ in THF (Scheme 3). Rearrangement of 13 afforded trifluoroacetamide 14 as the mixture of diastereoizomers ( $d e=19 \%$ ) (Scheme 3). In order to determine the best reaction conditions, a series of the thermally and microwave accelerated rearrangements of imidate $\mathbf{1 3}$ was performed. Studies showed that microwave irradiation accelerated of the rearrangement $\mathbf{1 3} \rightarrow \mathbf{1 4 a}, \mathbf{b}$ (24 times) in comparison with conventional thermal conditions (Table 2 , entry 4) without any improvement in the stereoselectivity. Extension of the reaction time led to the decomposition of product 14.

\section{Conclusions}

In summary, a remarkable acceleration of the Overman rearrangemet of allylic trihaloimidates to the corresponding allylic trihaloamides was observed using microwave irradiation conditions. The $[3,3]$-sigmatropic rearrangement carried out under conventional conditions (reflux temperature of the solvent) required long reaction times and produced moderate yields, usually a result of connected with the decomposition of starting materials. This paper demonstrates the practical usability of microwave acceterated thermal Overman rearrangement for the synthesis of various amides.

\section{Experimental}

All commercially available reagents were used without further purification and solvents were dried according to standard procedures. Column chromatography was carried out on Silica Gel 60 (Merck, 
0.040-0.063 mm, 230-400 mesh). Analytical thin-layer chromatography (TLC) was performed on Merck silica gel $60 \mathrm{~F}_{254}$ analytical plates; detection was carried out with either UV (254 nm), or spraying with a solution of phosphomolybdic acid, and with a basic solution of $\mathrm{KMnO}_{4}$, with subsequent heating. NMR spectra were recorded at room temperature on a Varian Mercury Plus 400 FT NMR spectrometer $\left({ }^{1} \mathrm{H}\right.$ at $400.13 \mathrm{MHz}$ and ${ }^{13} \mathrm{C}$ at $100.6 \mathrm{MHz}$ ), in $\mathrm{CDCl}_{3}$ as the solvent (unless otherwise noted) with tetramethylsilane as internal reference. For those fully assigned ${ }^{1} \mathrm{H}$ - and ${ }^{13} \mathrm{C}$ NMR spectra standard NMR (COSY, DEPT, HSQC) experiments were conducted. Optical rotations were measured with a P3002 Krüss polarimeter in chloroform at $25{ }^{\circ} \mathrm{C}$. All moisture-sensitive reactions were performed under a nitrogen atmosphere. Microwave experiments were conducted using a focused microwave system (CEM Discover). Reactions were performed in a glass vessel (10 $\mathrm{mL})$ sealed with a septum. At the end of the reaction the vessels together with their contents were cooled rapidly using a stream of compressed air. The melting points were determined on the Kofler block and are uncorrected.

\section{General procedure for preparation of trichloroacetimidates}

To a solution of allyl alcohol in dry dichloromethane were added 1,8-diazabicyclo[5,4,0]undec-7ene (DBU, $2 \mathrm{eq}$ ) and trichloroacetonitrile $(2 \mathrm{eq})$ at $0{ }^{\circ} \mathrm{C}$. The reaction mixture was stirred at $0{ }^{\circ} \mathrm{C}$ for 1 $\mathrm{h}$. The insoluble material was removed by filtration and the filtrate was concentrated under reduced pressure to give a residue, which was purified by chromatography on silica gel (cyclohexane-ethyl acetate) to afford corresponding imidates $\mathbf{1 a - 1 d , ~ 6 , ~ 7 , ~} 8$.

O-Allyl-2,2,2-trichloroacetimidate: Allyl alcohol (0.50 g, $8.61 \mathrm{mmol})$, DBU (2.57 mL, $17.22 \mathrm{mmol})$, trichloroacetonitrile $(1.73 \mathrm{~mL}, 17.22 \mathrm{mmol})$ in $\mathrm{CH}_{2} \mathrm{Cl}_{2}(20 \mathrm{~mL})$ afforded after flash chromatography (cyclohexane-ethyl acetate, 10:1) compound $1 \mathrm{a}(1.56 \mathrm{~g}, 89.5 \%)$ as a colorless oil; ${ }^{1} \mathrm{H}-\mathrm{NMR}: \delta 4.81$ $\left(2 \mathrm{H}, \mathrm{m}, \mathrm{CH}_{2}\right), 5.31\left(1 \mathrm{H}, \mathrm{dd}, J=10.5 \mathrm{~Hz}, J=1.3 \mathrm{~Hz}, \mathrm{CH}_{2}=\right), 5.44(1 \mathrm{H}$, ddd, $J=17.2 \mathrm{~Hz}, J=3.1 \mathrm{~Hz}, J=1.5$ $\left.\mathrm{Hz}, \mathrm{CH}_{2}=\right), 6.03(1 \mathrm{H}, \mathrm{ddd}, J=17.2 \mathrm{~Hz}, J=10.5 \mathrm{~Hz}, J=5.4 \mathrm{~Hz}, \mathrm{CH}=), 8.32(1 \mathrm{H}, \mathrm{bs}, \mathrm{NH}) ;{ }^{13} \mathrm{C}-\mathrm{NMR}: \delta$ 69.6, 109.7, 118.5, 131.4, 162.5; Anal. Calcd. for $\mathrm{C}_{5} \mathrm{H}_{6} \mathrm{Cl}_{3} \mathrm{NO}$ (202.47): C 29.66, H 2.99, N 6.91; found $\mathrm{C} 29.53, \mathrm{H} 2.87, \mathrm{~N} 6.74$. The procedure and ${ }^{1} \mathrm{H}-\mathrm{NMR}$ spectroscopic data were previously reported [10]. ${ }^{13} \mathrm{C}-\mathrm{NMR}$ data have not been reported before [10].

O-(But-3-en-2-yl)-2,2,2-trichloroacetimidate (1b): But-3-en-2-ol (0.50 g, $6.93 \mathrm{mmol}), \mathrm{DBU}$ (1.45 mL, $9.70 \mathrm{mmol}, 1.4 \mathrm{eq})$, trichloroacetonitrile $(1.04 \mathrm{~mL}, 10.4 \mathrm{mmol}, 1.5 \mathrm{eq})$ in $\mathrm{CH}_{2} \mathrm{Cl}_{2}(25 \mathrm{~mL})$ afforded (1.30 g, 87\%) of compound $\mathbf{1 b}$ after flash chromatography (cyclohexane-ethyl acetate, 5:1) as a pale yellow oil; ${ }^{1} \mathrm{H}-\mathrm{NMR}$ : $\delta 1.44\left(3 \mathrm{H}, \mathrm{d}, J=6.5 \mathrm{~Hz}, \mathrm{CH}_{3}\right), 5.20\left(1 \mathrm{H}, \mathrm{d}, J=10.6 \mathrm{~Hz}, \mathrm{H}_{4}\right), 5.36(1 \mathrm{H}, \mathrm{d}, J=17.3$ $\left.\mathrm{Hz}, \mathrm{H}_{4}\right), 5.49\left(1 \mathrm{H}, \mathrm{m}, \mathrm{H}_{3}\right), 5.94\left(1 \mathrm{H}, \mathrm{m}, \mathrm{H}_{2}\right), 8.29(1 \mathrm{H}, \mathrm{bs}, \mathrm{NH}) ;{ }^{13} \mathrm{C}-\mathrm{NMR}: \delta 19.4,75.7,91.8,115.9$, 136.8, 161.8.; Anal. Calcd. for $\mathrm{C}_{6} \mathrm{H}_{8} \mathrm{Cl}_{3} \mathrm{NO}$ (216.49): C 33.29, H 3.72, N 6.47; found C 33.10, H 3.45, $\mathrm{N}$ 6.28. The procedure and ${ }^{1} \mathrm{H}-\mathrm{NMR}$ spectroscopic data have been reported [18]. ${ }^{13} \mathrm{C}-\mathrm{NMR}$ data have not previously been reported [18].

2,2,2-Trichloro-O-(hept-1-en-3-yl)acetimidate (1c): Hept-1-en-3-ol (0.50 g, 4.38 mmol), DBU (1.31 $\mathrm{mL}, 8.76 \mathrm{mmol})$, trichloroacetonitrile $(0.88 \mathrm{~mL}, 8.76 \mathrm{mmol})$ in $\mathrm{CH}_{2} \mathrm{Cl}_{2}(20 \mathrm{~mL})$ afforded after flash 
chromatography (cyclohexane-ethyl acetate, 10:1) compound $1 \mathrm{c}(1.11 \mathrm{~g}, 98 \%)$ as a pale yellow oil; ${ }^{1} \mathrm{H}-\mathrm{NMR}: \delta 0.90\left(3 \mathrm{H}, \mathrm{t}, J=6.9 \mathrm{~Hz}, \mathrm{CH}_{3}\right), 1.38\left(4 \mathrm{H}, \mathrm{m}, 2 \times \mathrm{CH}_{2}\right), 1.74\left(2 \mathrm{H}, \mathrm{m}, \mathrm{CH}_{2}\right), 5.21(1 \mathrm{H}$, dd, $\left.J_{2,1}=10.6 \mathrm{~Hz}, J_{1,1}=0.7 \mathrm{~Hz}, \mathrm{H}_{1}\right), 5.36\left(2 \mathrm{H}, \mathrm{m}, \mathrm{H}_{1}, \mathrm{H}_{3}\right), 5.80\left(1 \mathrm{H}, \mathrm{m}, \mathrm{H}_{2}\right), 8.27(1 \mathrm{H}, \mathrm{s}, \mathrm{NH}) ;{ }^{13} \mathrm{C}-\mathrm{NMR}: \delta$ 14.2, 22.6, 27.3, 34.0, 79.7 92.1, 116.8, 135.8, 162.2; Anal. Calcd for $\mathrm{C}_{9} \mathrm{H}_{14} \mathrm{Cl}_{3} \mathrm{NO}_{2}$ (258.57): C 41.76, $\mathrm{H}$ 5.41, N 5.41; found C 41.65, H 5.21, N 5.33. The procedure and ${ }^{1} \mathrm{H}-\mathrm{NMR}$ spectroscopic data were reported [10]. ${ }^{13} \mathrm{C}-\mathrm{NMR}$ data have not previously been reported [10].

2,2,2-Trichloro-O-(oct-1-en-3-yl)acetimidate (1d): Oct-1-en-3-ol (0.50 g, 3.90 mmol), DBU (1.17 mL, $7.8 \mathrm{mmol})$, trichloroacetonitrile $(0.78 \mathrm{~mL}, 7.80 \mathrm{mmol})$ in $\mathrm{CH}_{2} \mathrm{Cl}_{2}(20 \mathrm{~mL})$ afforded after flash chromatography (cyclohexane-ethyl acetate, 10:1) compound $\mathbf{1 d}(0.90 \mathrm{~g}, 85 \%)$ as a pale yellow oil; ${ }^{1} \mathrm{H}-\mathrm{NMR}: \delta 0.88\left(3 \mathrm{H}, \mathrm{t}, J=7.1 \mathrm{~Hz}, \mathrm{CH}_{3}\right), 1.29-1.47\left(6 \mathrm{H}, \mathrm{m}, 3 \times \mathrm{CH}_{2}\right), 1.65-1.82\left(2 \mathrm{H}, \mathrm{m}, \mathrm{CH}_{2}\right), 5.21$ $\left(1 \mathrm{H}, \mathrm{m}, \mathrm{H}_{1}\right), 5.30\left(2 \mathrm{H}, \mathrm{m}, \mathrm{H}_{1}, \mathrm{H}_{3}\right), 5.86\left(1 \mathrm{H}, \mathrm{m}, \mathrm{H}_{2}\right), 8.26(1 \mathrm{H}, \mathrm{bs}, \mathrm{NH}) ;{ }^{13} \mathrm{C}-\mathrm{NMR}: \delta 14.2,79.7,22.7$, 24.8, 31.7, 34.3, 92.1, 116.7, 136.0, 162.2; Anal. Calcd. for $\mathrm{C}_{10} \mathrm{H}_{16} \mathrm{Cl}_{3} \mathrm{NO}$ (272.60): $\mathrm{C} 44.06, \mathrm{H}$ 5.91, $\mathrm{N}$ 5.14; found C 43.95, H 5.77, N 5.01.

tert-Butyl N-[(3S,4E)-6-(trichloroacetimidyloxy)hex-4-en-3-yl]carbamate (6): Compound 3 (0.30 g, $1.393 \mathrm{mmol})$, DBU $(0.42 \mathrm{~mL}, 2.79 \mathrm{mmol})$, trichloroacetonitrile $(0.28 \mathrm{~mL}, 2.79 \mathrm{mmol})$ in $\mathrm{CH}_{2} \mathrm{Cl}_{2}(15$ $\mathrm{mL}$ ) afforded after flash chromatography (cyclohexane-ethyl acetate, 3:1) compound 6 (0.40 g, 80\%) as a colorless oil; ${ }^{1} \mathrm{H}-\mathrm{NMR}: \delta 0.92\left(3 \mathrm{H}, \mathrm{t}, J=7.4 \mathrm{~Hz}, \mathrm{CH}_{3}\right), 1.44\left(9 \mathrm{H}, \mathrm{s}, 3 \times \mathrm{CH}_{3}\right), 1.53\left(2 \mathrm{H}, \mathrm{m}, \mathrm{CH}_{2}\right)$, $4.08\left(1 \mathrm{H}, \mathrm{m}, \mathrm{H}_{3}\right), 4.45(1 \mathrm{H}, \mathrm{bs}, \mathrm{NH}), 4.77\left(2 \mathrm{H}, \mathrm{m}, \mathrm{H}_{6}\right), 5.79\left(2 \mathrm{H}, \mathrm{m}, \mathrm{H}_{4}, \mathrm{H}_{5}\right), 8.29(1 \mathrm{H}, \mathrm{bs}, \mathrm{NH}) ;{ }^{13} \mathrm{C}-$ NMR: $\delta$ 10.1, 28.2, $28.4(3 \mathrm{x}), 53.1,68.9,79.4,123.4,135.8,155.4,162.5$; Anal. Calcd. for $\mathrm{C}_{13} \mathrm{H}_{21} \mathrm{Cl}_{3} \mathrm{~N}_{2} \mathrm{O}_{3}$ (359.68): C 43.37, $\mathrm{H}$ 5.83, N 7.78; found $\mathrm{C} 43.01, \mathrm{H}$ 5.64, N 7.64. ${ }^{1} \mathrm{H}$ and ${ }^{13} \mathrm{C}-\mathrm{NMR}$ spectroscopic data have not previously been reported [12].

tert-Butyl N-[(3S,4E)-6-(trichloroacetimidyloxy)-2-methylhex-4-en-3-yl]carbamate (7): Compound 4 $(0.10 \mathrm{~g}, 0.436 \mathrm{mmol}), \mathrm{DBU}(0.13 \mathrm{~mL}, 0.87 \mathrm{mmol})$, trichloroacetonitrile $(0.087 \mathrm{~mL}, 0.87 \mathrm{mmol})$ in $\mathrm{CH}_{2} \mathrm{Cl}_{2}(10 \mathrm{~mL})$ afforded after flash chromatography (cyclohexane-ethyl acetate, $\left.3: 1\right)$ compound 7 $(0.12 \mathrm{~g}, 74 \%)$ as white crystals; m.p. $42-43{ }^{\circ} \mathrm{C} ;{ }^{1} \mathrm{H}-\mathrm{NMR}: \delta 0.89\left(6 \mathrm{H}, \mathrm{m}, 2 \times \mathrm{CH}_{3}\right), 1.44(9 \mathrm{H}, \mathrm{s}, 3 \mathrm{x}$ $\left.\mathrm{CH}_{3}\right), 1.78(1 \mathrm{H}, \mathrm{m}, \mathrm{CH}), 4.04\left(1 \mathrm{H}, \mathrm{m}, \mathrm{H}_{3}\right), 4.52(1 \mathrm{H}, \mathrm{m}, \mathrm{NH}), 4.80\left(2 \mathrm{H}, \mathrm{m}, \mathrm{H}_{6}\right), 5.79\left(2 \mathrm{H}, \mathrm{m}, \mathrm{H}_{4}, \mathrm{H}_{5}\right)$, $8.30(1 \mathrm{H}, \mathrm{bs}, \mathrm{NH}) ;{ }^{13} \mathrm{C}-\mathrm{NMR}: \delta 18.1,18.7,28.4$, $(3 \mathrm{x} \mathrm{C}), 32.4,56.9,68.9,79.4,91.4,123.9,134.5$, 155.5, 162.4; Anal. Calcd. for $\mathrm{C}_{14} \mathrm{H}_{23} \mathrm{Cl}_{3} \mathrm{~N}_{2} \mathrm{O}_{3}$ (373.71): C 44.99, $\mathrm{H}$ 6.20, N 7.49; found $\mathrm{C} 44.78, \mathrm{H}$ 6.05, N 7.21. ${ }^{1} \mathrm{H}$ - and ${ }^{13} \mathrm{C}-\mathrm{NMR}$ spectroscopic data have not previously been reported [12].

O-[(4S,2E)-4-(tert-Butyldimethylsilyoxy)pent-2-enyl]-2,2,2-trichloroacetimidate (8): Compound $\mathbf{5}$ (0.35 g, $1.62 \mathrm{mmol})$, DBU $(0.48 \mathrm{~mL}, 3.24 \mathrm{mmol})$, trichloroacetonitrile $(0.325 \mathrm{~mL}, 3.24 \mathrm{mmol})$ in $\mathrm{CH}_{2} \mathrm{Cl}_{2}(18 \mathrm{~mL})$ afforded compound $8(0.50 \mathrm{~g}, 85.5 \%)$ as a colorless oil; ${ }^{1} \mathrm{H}-\mathrm{NMR}: \delta 0.05(3 \mathrm{H}, \mathrm{s}$, $\left.\mathrm{CH}_{3}\right), 0.06\left(3 \mathrm{H}, \mathrm{s}, \mathrm{CH}_{3}\right), 0.89\left(9 \mathrm{H}, \mathrm{s}, 3 \times \mathrm{CH}_{3}\right), 1.23\left(3 \mathrm{H}, \mathrm{d}, J=6.7 \mathrm{~Hz}, \mathrm{CH}_{3}\right), 4.35\left(1 \mathrm{H}, \mathrm{m}, \mathrm{H}_{4}\right), 4.77$ $\left(2 \mathrm{H}, \mathrm{m}, \mathrm{H}_{1}\right), 5.86\left(2 \mathrm{H}, \mathrm{m}, \mathrm{H}_{2}, \mathrm{H}_{3}\right), 8.28(1 \mathrm{H}, \mathrm{bs}, \mathrm{NH}) ;{ }^{13} \mathrm{C}-\mathrm{NMR}: \delta-4.8,-4.7,18.3,24.1,25.9$ (3 x C), 68.4, 69.1, 121.4, 139.8, 162.5; Anal. Calcd. for $\mathrm{C}_{13} \mathrm{H}_{24} \mathrm{Cl}_{3} \mathrm{NO}_{2} \mathrm{Si}$ (360.78): C 43.28, H 6.71, N 3.88; found $\mathrm{C} 43.17, \mathrm{H} 6.56, \mathrm{~N} 3.59$. The procedure and ${ }^{1} \mathrm{H}-\mathrm{NMR}$ spectroscopic data were reported before [14]. ${ }^{13} \mathrm{C}-\mathrm{NMR}$ data have not previously been reported [14]. 


\section{General procedure for Overman rearrangement}

Conventional method (Procedure A): To a solution of imidates in dry solvent (see Tables 1, 2) was added anhydrous $\mathrm{K}_{2} \mathrm{CO}_{3}(1.1 \mathrm{eq})$. The reaction mixture was heated (for temperatures see Tables 1, 2). The solvent was evaporated under reduced pressure and chromatography of the residue on the silica gel (cyclohexane-ethyl acetate) afforded corresponding amides 2a-2f, 9-11, 14 (Tables 1, 2). (B1)

Microwave-assisted synthesis (Procedure B): To a solution of the corresponding imidate in $o$-xylene in a $10 \mathrm{~mL}$ glass pressure microwave tube equipped with a magnetic stirrer bar was added anhydrous $\mathrm{K}_{2} \mathrm{CO}_{3}(1.1 \mathrm{eq})$ and the tube was closed with a silicon septum. The reaction mixture was subjected to microwave irradiation (power: $300 \mathrm{~W}$; for temperatures, reaction times and yields see Tables 1, 2). The solvent was removed under reduced pressure and the residue was purified by flash chromatography on silica gel (cyclohexane-ethyl acetate) to give amides 2a-2f, 9-11, 14 (Tables 1, 2). (B2)

$N$-Allyl-2,2,2-trichloroacetamide (2a): Following general procedure A, $1 \mathbf{a}(0.30 \mathrm{~g}, 1.48 \mathrm{mmol}), \mathrm{K}_{2} \mathrm{CO}_{3}$ $(0.23 \mathrm{~g}, 1.63 \mathrm{mmol})$ in $o$-xylene $(3 \mathrm{~mL})$ afforded after flash chromatography (cyclohexane-ethyl acetate, 10:1) compound 2a (0.195 g, 65\%). 2a: white crystals; m.p. 28 - $32{ }^{\circ} \mathrm{C}$ (Ref. [10] m.p. $28-31$ $\left.{ }^{\circ} \mathrm{C}\right)$; ${ }^{1} \mathrm{H}-\mathrm{NMR}: \delta 3.99-4.02\left(2 \mathrm{H}, \mathrm{m}, \mathrm{CH}_{2}\right), 5.24-5.32\left(2 \mathrm{H}, \mathrm{m}, \mathrm{CH}_{2}=\right), 5.84-5.93(1 \mathrm{H}, \mathrm{m}, \mathrm{CH}=), 6.78(1 \mathrm{H}$, bs, $\mathrm{NH}) ;{ }^{13} \mathrm{C}-\mathrm{NMR}: \delta$ 43.6, 92.5, 117.8, 132.2, 161.8; Anal. Calcd. for $\mathrm{C}_{5} \mathrm{H}_{6} \mathrm{Cl}_{3} \mathrm{NO}$ (202.46): C 29.66, H 2.98, N 7.90; found C 29.59, H 2.83, N 7.75. The procedure and ${ }^{1} \mathrm{H}-\mathrm{NMR}$ spectroscopic data were reported [10]. ${ }^{13} \mathrm{C}-\mathrm{NMR}$ data have not previously been reported [10].

$N$-[(E)-But-2-enyl]-2,2,2-trichloroacetamide (2b): Following general procedure A, $1 \mathbf{b}(0.10 \mathrm{~g}, 0.462$ $\mathrm{mmol}), \mathrm{K}_{2} \mathrm{CO}_{3}(70.2 \mathrm{mg}, 0.508 \mathrm{mmol})$ in $o$-xylene $(2 \mathrm{~mL})$ afforded after flash chromatography (cyclohexane-ethyl acetate, 10:1) compound $2 \mathbf{b}(0.093 \mathrm{~g}, 93 \%)$. Following general procedure B, 1b $(0.30 \mathrm{~g}, 1.386 \mathrm{mmol}), \mathrm{K}_{2} \mathrm{CO}_{3}(0.21 \mathrm{~g}, 1.525 \mathrm{mmol})$ in $o$-xylene $(5 \mathrm{~mL})$ afforded after flash chromatography (cyclohexane-ethyl acetate, 10:1) compound $\mathbf{2 b}(0.18 \mathrm{~g}, 60 \%)$. 2b: white crystals; m.p. $27-29{ }^{\circ} \mathrm{C}$ (Ref. [10] m.p. $\left.28-29{ }^{\circ} \mathrm{C}\right)$; ${ }^{1} \mathrm{H}-\mathrm{NMR}$ : $\delta 1.72\left(3 \mathrm{H}, \mathrm{d}, J=6.5 \mathrm{~Hz}, \mathrm{CH}_{3}\right), 3.91(2 \mathrm{H}, \mathrm{m}$, $\left.\mathrm{CH}_{2}\right), 5.50(1 \mathrm{H}, \mathrm{m}, \mathrm{CH}=), 5.74(1 \mathrm{H}, \mathrm{m}, \mathrm{CH}=), 6.68(1 \mathrm{H}, \mathrm{bs}, \mathrm{NH}) ;{ }^{13} \mathrm{C}-\mathrm{NMR}: \delta 17.7,43.3,109.7$, 124.8, 130.3, 161.6; Anal. Calcd. for $\mathrm{C}_{6} \mathrm{H}_{8} \mathrm{Cl}_{3} \mathrm{NO}$ (216.49): C 33.26, H 3.72, N 6.47; found C 33.14, $\mathrm{H}$ 3.55, N 6.38. The procedure, ${ }^{1} \mathrm{H}-\mathrm{NMR}$ and ${ }^{13} \mathrm{C}-\mathrm{NMR}$ data spectroscopic data have been reported [18].

2,2,2-Trichloro- $N$ - $[(E)$-hept-2-enyl] acetamide (2c): Following general procedure A, 1c $(0.20 \mathrm{~g}, 0.773$ $\mathrm{mmol}), \mathrm{K}_{2} \mathrm{CO}_{3}(0.117 \mathrm{~g}, 0.85 \mathrm{mmol})$ in $o$-xylene $(2 \mathrm{~mL})$ afforded after flash chromatography (cyclohexane-ethyl acetate, 10:1) compound 2c (0.18 g, 90\%). Following general procedure B, 1c $(0.40 \mathrm{~g}, 1.55 \mathrm{mmol}), \mathrm{K}_{2} \mathrm{CO}_{3}(0.236 \mathrm{~g}, 1.71 \mathrm{mmol})$ in $o$-xylene $(4 \mathrm{~mL})$ afforded after flash chromatography (cyclohexane-ethyl acetate, 10:1) compound $2 \mathrm{c}(0.30 \mathrm{~g}, 75 \%)$. 2c: a colorless oil; ${ }^{1} \mathrm{H}-$ NMR: $\delta 0.90\left(3 \mathrm{H}, \mathrm{t}, J=7.1 \mathrm{~Hz}, \mathrm{CH}_{3}\right), 1.28-1.39(4 \mathrm{H}, \mathrm{m}, 2 \mathrm{x} \mathrm{CH}), 2.02-2.07\left(2 \mathrm{H}, \mathrm{m}, \mathrm{CH}_{2}\right), 3.92(2 \mathrm{H}$, $\left.\mathrm{m}, \mathrm{CH}_{2}\right)$; 5.44-5.51 $(1 \mathrm{H}, \mathrm{m}, \mathrm{CH}=), 5.68-5.76(1 \mathrm{H}, \mathrm{m}, \mathrm{CH}=), 6.67(1 \mathrm{H} ; \mathrm{bs}, \mathrm{NH}) ;{ }^{13} \mathrm{C}-\mathrm{NMR}: \delta 14.1$, 22.4, 31.3, 32.1, 43.6, 92.8, 123.7, 135.9, 161.8; Anal. Calcd. for $\mathrm{C}_{9} \mathrm{H}_{14} \mathrm{Cl}_{3} \mathrm{NO}_{2}$ (258.57): $\mathrm{C} 41.77, \mathrm{H}$ 5.45, N 5.41; found $\mathrm{C} 41.63, \mathrm{H} 5.24, \mathrm{~N} 5.35$. The procedure and ${ }^{1} \mathrm{H}-\mathrm{NMR}$ spectroscopic data were reported before [10]. ${ }^{13} \mathrm{C}-\mathrm{NMR}$ data have not previously been reported [10]. 
(E)-2,2,2-Trichloro-N-(oct-2-enyl)acetamide (2d): Following general procedure A, 1d (0.40 g, 1.47 $\mathrm{mmol}), \mathrm{K}_{2} \mathrm{CO}_{3}(0.224 \mathrm{~g}, 1.62 \mathrm{mmol})$ in $o$-xylene $(4 \mathrm{~mL})$ afforded after flash chromatography (cyclohexane-ethyl acetate, 10:1) compound 2d (0.37 g, 92.5\%). Following general procedure B, 1d $(0.40 \mathrm{~g}, 1.47 \mathrm{mmol}), \mathrm{K}_{2} \mathrm{CO}_{3}(0.224 \mathrm{~g}, 1.62 \mathrm{mmol})$ in $o$-xylene $(4 \mathrm{~mL})$ afforded after flash chromatography (cyclohexane-ethyl acetate, 10:1) compound $\mathbf{2 d}(0.39 \mathrm{~g}, 97.5 \%)$. 2d: a colorless oil; ${ }^{1} \mathrm{H}-\mathrm{NMR}: \delta 0.87\left(3 \mathrm{H}, \mathrm{t}, J=7.0 \mathrm{~Hz}, \mathrm{CH}_{3}\right), 1.26-1.40\left(6 \mathrm{H}, \mathrm{m}, 3 \times \mathrm{CH}_{2}\right), 2.04\left(2 \mathrm{H}, \mathrm{q}, J=7.0 \mathrm{~Hz}, \mathrm{CH}_{2}\right)$, $3.93\left(2 \mathrm{H}, \mathrm{t}, J=5.9 \mathrm{~Hz}, \mathrm{CH}_{2}\right), 5.46(1 \mathrm{H}, \mathrm{m}, \mathrm{CH}=), 5.73(1 \mathrm{H}, \mathrm{m}, \mathrm{CH}=), 6.69(1 \mathrm{H}, \mathrm{bs}, \mathrm{NH}) ;{ }^{13} \mathrm{C}-\mathrm{NMR}: \delta$ 14.6, 22.7, 28.8, 31.6, 32.4, 43.5, 92.8, 123.6, 135.8, 161.7; Anal. Calcd. for $\mathrm{C}_{10} \mathrm{H}_{16} \mathrm{Cl}_{3} \mathrm{NO}$ (272.60): C 44.02, H 5.92, N 5.13; found C 43.90, H 5.84, N 5.04.

2,2,2-Trichloro- $\mathrm{N}$-(2-methylbut-3-en-2-yl)acetamide (2e): Following general procedure A, $1 \mathrm{e}(0.3 \mathrm{~g}$, $1.30 \mathrm{mmol}), \mathrm{K}_{2} \mathrm{CO}_{3}(0.198 \mathrm{~g}, 1.43 \mathrm{mmol})$ in $o$-xylene $(3 \mathrm{~mL})$ afforded after flash chromatography (cyclohexane-ethyl acetate, 10:1) compound 2e (0.25 g, 83\%). Following general procedure B, 1e $(0.5 \mathrm{~g}, 2.17 \mathrm{mmol}), \mathrm{K}_{2} \mathrm{CO}_{3}(0.33 \mathrm{~g}, 2.39 \mathrm{mmol})$ in $o$-xylene $(5 \mathrm{~mL})$ afforded after flash chromatography (cyclohexane-ethyl acetate, 10:1) compound $2 \mathrm{e}(0.40 \mathrm{~g}, 80 \%)$. 2e: white crystals; m.p. $48-50{ }^{\circ} \mathrm{C}$ (Ref. [11] m.p. $\left.49-50{ }^{\circ} \mathrm{C}\right) ;{ }^{1} \mathrm{H}-\mathrm{NMR}: \delta 1.54\left(6 \mathrm{H}, \mathrm{s}, 2 \times \mathrm{CH}_{3}\right), 5.17\left(2 \mathrm{H}, \mathrm{m}, \mathrm{H}_{4}\right), 6.00\left(1 \mathrm{H}, \mathrm{m}, \mathrm{H}_{3}\right), 6.59(1 \mathrm{H}$, bs, $\mathrm{NH}) ;{ }^{13} \mathrm{C}-\mathrm{NMR}: \delta 26.5(2 \times \mathrm{C}), 56.1,93.4,113.3,142.1,160.4$; Anal. Calcd. for $\mathrm{C}_{7} \mathrm{H}_{10} \mathrm{Cl}_{3} \mathrm{NO}$ (230.52): C 36.43, H 4.32, N 6.07, found C 36.30, H 4.11, N 5.98. The ${ }^{1} \mathrm{H}-\mathrm{NMR}$ spectrum was previously reported [11].

2,2,2-Trichloro- $\mathrm{N}-[($ E)-pent-3-en-2-yl]acetamide (2f): Following general procedure A, $1 \mathbf{f}(0.10 \mathrm{~g}$, $0.434 \mathrm{mmol}), \mathrm{K}_{2} \mathrm{CO}_{3}(66 \mathrm{mg}, 0.48 \mathrm{mmol})$ in $o$-xylene $(2 \mathrm{~mL})$ afforded after flash chromatography (cyclohexane-ethyl acetate, 10:1) compound $\mathbf{2 f}$ (75 mg, 75\%). Following general procedure B, 1 f (66 $\mathrm{mg}, 0.26 \mathrm{mmol}), \mathrm{K}_{2} \mathrm{CO}_{3}(43.5 \mathrm{mg}, 0.315 \mathrm{mmol})$ in $o$-xylene $(1 \mathrm{~mL})$ afforded after flash chromatography (cyclohexane-ethyl acetate, 10:1) compound $2 \mathrm{f}$ (46 mg, 70\%) 2f: white crystals; m.p. $57-59{ }^{\circ} \mathrm{C}$ (Ref. [20] m.p. $\left.60{ }^{\circ} \mathrm{C}\right)$; ${ }^{1} \mathrm{H}-\mathrm{NMR}$ (DMSO [20]): $\delta 1.31\left(3 \mathrm{H}, \mathrm{d}, J=6.8 \mathrm{~Hz}, \mathrm{CH}_{3}\right), 1.71(3 \mathrm{H}$, $\left.\mathrm{m}, \mathrm{CH}_{3}\right), 4.43-4.50\left(1 \mathrm{H}, \mathrm{m}, \mathrm{H}_{2}\right), 5.43-5.49(1 \mathrm{H}, \mathrm{m}, \mathrm{CH}=), 5.65-5.75(1 \mathrm{H}, \mathrm{m}, \mathrm{CH}=), 6.52(1 \mathrm{H}, \mathrm{bs}, \mathrm{NH})$; ${ }^{13} \mathrm{C}-\mathrm{NMR}$ (DMSO [20]): $\delta$ 17.9, 20.4, 49.0, 93.0, 127.6, 130.76, 161.03; Anal. Calcd. for $\mathrm{C}_{7} \mathrm{H}_{10} \mathrm{Cl}_{3} \mathrm{NO}$ (230.52): C 36.44, H 4.37, N 6.07, found C 36.35, H 4.21, N 5.97.

Methyl (Z)-2,3,4-tri-O-benzyl-6,7-dideoxy-8-(trifluoroacetimidyloxy)- $\alpha$-D-gluco-oct-6-enpyranoside (13): To a suspension of $\mathrm{NaH}(0.09 \mathrm{~g}, 2.244 \mathrm{mmol}, 60 \%$ dispersion in mineral oil, freed of oil with anhydrous THF) in dry THF (3 mL) was added allylic alcohol 12 (1.0 g, $2.04 \mathrm{mmol})$ in dry THF (5 $\mathrm{mL}$ ) at $0{ }^{\circ} \mathrm{C}$. The reaction mixture was stirred at $0{ }^{\circ} \mathrm{C}$ for $10 \mathrm{~min}$ and then treated with gaseous trifluoroacetonitrile $(15 \mathrm{~g}, 0.158 \mathrm{~mol}$, prepared in situ by heating trifluoroacetamide $(4.57 \mathrm{~g}, 0.040$ $\mathrm{mol})$ and $\mathrm{P}_{2} \mathrm{O}_{5}(11.43 \mathrm{~g}, 0.102 \mathrm{~mol})$ for $2 \mathrm{~h}$ at $\left.150{ }^{\circ} \mathrm{C}\right)$. The solid was removed by filtration and solvent evaporated under reduced pressure. The residue was purified by chromatography on silica gel (hexaneethyl acetate, $3: 1)$ to afford $0.95 \mathrm{~g}(79.5 \%)$ of compound $\mathbf{1 3}$ as a pale yellow oil; ${ }^{1} \mathrm{H}-\mathrm{NMR}: \delta 3.27(1 \mathrm{H}$, $\left.\mathrm{dd}, J_{4,3}=9.6 \mathrm{~Hz}, J_{5,4}=9.1 \mathrm{~Hz}, \mathrm{H}_{4}\right), 3.40\left(3 \mathrm{H}, \mathrm{s}, \mathrm{OCH}_{3}\right), 3.52\left(1 \mathrm{H}, \mathrm{dd}, J_{3,2}=9.7 \mathrm{~Hz}, J_{2,1}=3.6 \mathrm{~Hz}, \mathrm{H}_{2}\right), 3.99$ $\left(1 \mathrm{H}, \mathrm{dd}, J_{3,2}=9.7 \mathrm{~Hz}, J_{4,3}=9.6 \mathrm{~Hz}, \mathrm{H}_{3}\right), 4.45\left(1 \mathrm{H}, \mathrm{ddd}, J_{5,4}=9.1 \mathrm{~Hz}, J_{6,5}=9.0 \mathrm{~Hz}, J_{7,5}=1.0, \mathrm{H}_{5}\right), 4.57(1 \mathrm{H}$, d, $\left.J=10.8 \mathrm{~Hz}, \mathrm{CH}_{2} \mathrm{Ph}\right), 4.57\left(1 \mathrm{H}, \mathrm{d}, J_{2,1}=3.6 \mathrm{~Hz}, \mathrm{H}_{1}\right), 4.67\left(1 \mathrm{H}, \mathrm{d}, J=12.1 \mathrm{~Hz}, \mathrm{CH}_{2} \mathrm{Ph}\right), 4.70(1 \mathrm{H}$, ddd, $\left.J_{8,8}=11.9 \mathrm{~Hz}, J_{8,7}=5.4 \mathrm{~Hz}, J_{8,6}=1.4 \mathrm{~Hz}, \mathrm{H}_{8}\right), 4.79\left(1 \mathrm{H}, \mathrm{d}, J=10.6 \mathrm{~Hz}, \mathrm{CH}_{2} \mathrm{Ph}\right), 4.80(1 \mathrm{H}, \mathrm{d}, J=12.1 \mathrm{~Hz}$, 
$\left.\mathrm{CH}_{2} \mathrm{Ph}\right), 4.82\left(1 \mathrm{H}, J=10.6 \mathrm{~Hz}, \mathrm{CH}_{2} \mathrm{Ph}\right), 4.96\left(1 \mathrm{H}, J=10.8 \mathrm{~Hz}, \mathrm{CH}_{2} \mathrm{Ph}\right), 5.01\left(1 \mathrm{H}\right.$, ddd, $J_{8,8}=11.9 \mathrm{~Hz}$, $\left.J_{8,7}=7.4 \mathrm{~Hz}, J_{8,6}=1.4 \mathrm{~Hz}, \mathrm{H}_{8}\right), 5.61\left(1 \mathrm{H}\right.$, dddd, $\left.J_{7,6}=11.2 \mathrm{~Hz}, J_{6,5}=9.0 \mathrm{~Hz}, J_{8,6}=1.4 \mathrm{~Hz}, J_{8,6}=1.4 \mathrm{~Hz}, \mathrm{H}_{6}\right)$ $5.81\left(1 \mathrm{H}\right.$, dddd, $\left.J_{7,6}=11.2 \mathrm{~Hz}, J_{8,7}=7.4 \mathrm{~Hz}, J_{8,7}=5.4 \mathrm{~Hz}, J_{7,5}=1.0 \mathrm{~Hz}_{2} \mathrm{H}_{7}\right), 7.22-7.37(15 \mathrm{H}, \mathrm{m}, \mathrm{Ph}), 8.20$ $(1 \mathrm{H}, \mathrm{bs}, \mathrm{NH}) ;{ }^{13} \mathrm{C}-\mathrm{NMR}: \delta 55.5,62.0,66.8,73.4,75.3,75.8,79.8,81.6,81.9,98.2,127.6,127.7$, $127.7,2 \times 127.8,127.9,2 \times 128.0,2 \times 128.1,2 \times 128.3,2 \times 128.4,2 \times 128.5,131.2,138.0,138.1,138.6$, 157.3, 157.7; Anal. Calcd. for $\mathrm{C}_{32} \mathrm{H}_{34} \mathrm{~F}_{3} \mathrm{NO}_{6}$ (585.63): C 65.63, H 5.85, N 2.39; found C 65.59, H 5.80, N 2.31 .

Methyl 2,3,4-tri-O-benzyl-6-[(trifluoroacetyl)amino]-7,8-dideoxy-D-glycero- $\alpha$-D-galacto-oct-7enpyranoside 14a, Methyl 2,3,4-tri-O-benzyl-6-[(trifluoroacetyl)amino]-7,8-dideoxy-L-glycero$\alpha$-D-galacto-oct-7-enpyranoside (14b): Following general procedure A, 13 (0.25 g, $0.43 \mathrm{mmol})$, $\mathrm{K}_{2} \mathrm{CO}_{3}(65.4 \mathrm{mg}, 0.47 \mathrm{mmol})$ in $o$-xylene $(2 \mathrm{~mL})$ afforded after flash chromatography (hexane-ethyl acetate, 9:1) compounds 14a and 14b (0.08 g, 32\%, see Table 4). Following general procedure B, 13 $(0.10 \mathrm{~g}, 0.171 \mathrm{mmol}), \mathrm{K}_{2} \mathrm{CO}_{3}(26 \mathrm{mg}, 0.188 \mathrm{mmol})$ in $o$-xylene $(2 \mathrm{~mL})$ afforded after flash chromatography (hexane-ethyl acetate, 15:1) compounds $\mathbf{1 4 a}$ and $\mathbf{1 4 b}(0.07 \mathrm{~g}, 70 \%$, see Table 2).

14a: a colorless oil; $[\alpha]_{\mathrm{D}}{ }^{25}=-19.6(c 0.23) ;{ }^{1} \mathrm{H}-\mathrm{NMR}: \delta 3.30\left(1 \mathrm{H}, \mathrm{dd}, J_{5,4}=10.0 \mathrm{~Hz}, J_{4,3}=9.2 \mathrm{~Hz}, \mathrm{H}_{5}\right)$, $3.33\left(3 \mathrm{H}, \mathrm{s}, \mathrm{OCH}_{3}\right), 3.49\left(1 \mathrm{H}, \mathrm{dd}, J_{3,2}=9.3 \mathrm{~Hz}, J_{2,1}=3.6 \mathrm{~Hz}, \mathrm{H}_{2}\right), 3.76\left(1 \mathrm{H}, \mathrm{dd}, J_{5,4}=10.0 \mathrm{~Hz}, J_{6,5}=1.4\right.$ $\left.\mathrm{Hz}, \mathrm{H}_{5}\right), 4.01\left(1 \mathrm{H}, \mathrm{dd}, J_{3,2}=9.3 \mathrm{~Hz}, J_{4,3}=9.2 \mathrm{~Hz}, \mathrm{H}_{3}\right), 4.50\left(1 \mathrm{H}, \mathrm{d}, J=10.1 \mathrm{~Hz}, \mathrm{CH}_{2} \mathrm{Ph}\right), 4.56(1 \mathrm{H}, \mathrm{d}$, $\left.J_{2,1}=3.6 . \mathrm{Hz}, \mathrm{H}_{1}\right), 4.66\left(1 \mathrm{H}, \mathrm{d}, J=12.1 \mathrm{~Hz}, \mathrm{CH}_{2} \mathrm{Ph}\right), 4.82\left(1 \mathrm{H}, \mathrm{d}, J=12.1 \mathrm{~Hz}, \mathrm{CH}_{2} \mathrm{Ph}\right), 4.84(1 \mathrm{H}, \mathrm{d}$, $\left.J=10.7 \mathrm{~Hz}, \mathrm{CH}_{2} \mathrm{Ph}\right), 4.90\left(1 \mathrm{H}, \mathrm{d}, J=10.1 \mathrm{~Hz}, \mathrm{CH}_{2} \mathrm{Ph}\right), 4.97\left(1 \mathrm{H}, \mathrm{ddd}, J_{6, \mathrm{NH}}=9.4 \mathrm{~Hz}, J_{7,6}=5.4 \mathrm{~Hz}\right.$, $\left.J_{6,5}=1.4 \mathrm{~Hz}, \mathrm{H}_{6}\right), 5.01\left(1 \mathrm{H}, \mathrm{d}, J=10.7 \mathrm{~Hz}, \mathrm{CH}_{2} \mathrm{Ph}\right), 5.23\left(1 \mathrm{H}, \mathrm{dd}, J_{8 c i s, 7}=10.3 \mathrm{~Hz}, J_{8 c i s, 8 \text { trans }}=1.6 \mathrm{~Hz}\right.$, $\left.\mathrm{H}_{8 c i s}\right), 5.23\left(1 \mathrm{H}, \mathrm{dd}, J_{8 \text { trans }, 7}=17.1 \mathrm{~Hz}, J_{8 \text { trans }, 8 c i s}=1.6 \mathrm{~Hz}, \mathrm{H}_{8 \text { trans }}\right), 5.81\left(1 \mathrm{H}\right.$, ddd, $J_{8 \text { trans }, 7}=17.1 \mathrm{~Hz}$, $\left.J_{8 c i s, 7}=10.3 \mathrm{~Hz}, J_{7,6}=5.4 \mathrm{~Hz}, \mathrm{H}_{7}\right), 6.71\left(1 \mathrm{H}, \mathrm{d}, J_{6, \mathrm{NH}}=9.4 \mathrm{~Hz}, \mathrm{NH}\right), 7.27-7.39(15 \mathrm{H}, \mathrm{m}, \mathrm{Ph}) ;{ }^{13} \mathrm{C}-\mathrm{NMR}: \delta$ $50.8,55.4,71.0,73.6,75.6,75.9,78.0,80.0,81.8,98.1,117.2,127.8,2 \times 128.0,4 \times 128.1,2 \times 128.4$, $2 \times 128.5,4 \times 128.6,133.8,137.4,137.9,138.2$, 156.6, 157.0; Anal. Calcd. for $\mathrm{C}_{32} \mathrm{H}_{34} \mathrm{~F}_{3} \mathrm{NO}_{6}(585.63)$ : C 65.63, H 5.85, N 2.39; found C 65.56, H 5.79, N 2.32.

14b: a colorless oil; $[\alpha]_{\mathrm{D}}{ }^{25}=+30.5(c 0.19) ;{ }^{1} \mathrm{H}-\mathrm{NMR}: \delta 3.35\left(3 \mathrm{H}, \mathrm{s}, \mathrm{OCH}_{3}\right), 3.41\left(1 \mathrm{H}, \mathrm{dd}, J_{5,4}=10.0\right.$ $\left.\mathrm{Hz}, J_{4,3}=8.9 \mathrm{~Hz}, \mathrm{H}_{4}\right), 3.48\left(1 \mathrm{H}, \mathrm{dd}, J_{3,2}=9.6 \mathrm{~Hz}, J_{2,1}=3.5 \mathrm{~Hz}, \mathrm{H}_{2}\right), 3.81\left(1 \mathrm{H}, \mathrm{dd}, J_{5,4}=10.0 \mathrm{~Hz}, J_{6,5}=2.7\right.$ $\left.\mathrm{Hz}, \mathrm{H}_{5}\right), 4.01\left(1 \mathrm{H}, \mathrm{dd}, J_{3,2}=9.6 \mathrm{~Hz}, J_{4,3}=8.9 \mathrm{~Hz}, \mathrm{H}_{3}\right), 4.57\left(1 \mathrm{H}, J_{2,1}=3.5 \mathrm{~Hz}, \mathrm{H}_{1}\right), 4.61(1 \mathrm{H}, \mathrm{d}, J=11.1$ $\left.\mathrm{Hz}, \mathrm{CH}_{2} \mathrm{Ph}\right), 4.65\left(1 \mathrm{H}, \mathrm{d}, J=12.1 \mathrm{~Hz}, \mathrm{CH}_{2} \mathrm{Ph}\right), 4.78\left(1 \mathrm{H}, \mathrm{d}, J=11.1 \mathrm{~Hz}, \mathrm{CH}_{2} \mathrm{Ph}\right), 4.81(1 \mathrm{H}, \mathrm{d}, J=12.1$ $\left.\mathrm{Hz}, \mathrm{CH}_{2} \mathrm{Ph}\right), 4.89\left(1 \mathrm{H}, \mathrm{ddd}, J_{6, \mathrm{NH}}=9.0 \mathrm{~Hz}, J_{7,6}=8.2 \mathrm{~Hz}, J_{6,5}=2.7 \mathrm{~Hz}, \mathrm{H}_{6}\right), 4.93(1 \mathrm{H}, \mathrm{d}, J=10.8 \mathrm{~Hz}$, $\left.\mathrm{CH}_{2} \mathrm{Ph}\right), 4.99\left(1 \mathrm{H}, \mathrm{d}, J=10.8 \mathrm{~Hz}, \mathrm{CH}_{2} \mathrm{Ph}\right), 5.25\left(1 \mathrm{H}, \mathrm{dd}, J_{8 \text { trans }, 7}=17.1 \mathrm{~Hz}, J_{8 \text { trans }, 8 c i s}=1.0 \mathrm{~Hz}, \mathrm{H}_{8 \text { trans }}\right)$, $5.29\left(1 \mathrm{H}, \mathrm{dd}, J_{8 c i s, 7}=10.3 \mathrm{~Hz}, J_{8 t r a n s, 8 c i s}=1.0 \mathrm{~Hz}, \mathrm{H}_{8 c i s}\right), 5.71\left(1 \mathrm{H}, \mathrm{ddd}, J_{8 t r a n s, 7}=17.1 \mathrm{~Hz}, J_{8 c i s, 7}=10.3 \mathrm{~Hz}\right.$, $\left.J_{7,6}=8.2 \mathrm{~Hz}, \mathrm{H}_{7}\right), 6.70\left(1 \mathrm{H}, \mathrm{d}, J_{6, \mathrm{NH}}=9.0 \mathrm{~Hz}, \mathrm{NH}\right), 7.27-7.39(15 \mathrm{H}, \mathrm{m}, \mathrm{Ph}) ;{ }^{13} \mathrm{C}-\mathrm{NMR}: \delta 52.4,55.5$, 71.6, 73.8, 74.7, 76.0, 77.8, 80.1, 82.1, 98.5, 121.4, 2x127.8, 128.0, 128.1, 2x128.2, 3x128.3, 2x128.7, $4 \times 128.8,131.1,2 \times 138.1,138.6,156.3,156.7$; Anal. Calcd. for $\mathrm{C}_{32} \mathrm{H}_{34} \mathrm{~F}_{3} \mathrm{NO}_{6}$ (585.63): $\mathrm{C} 65.63, \mathrm{H}$ 5.85, N 2.39; found C 65.53, H 5.76, N 2.45 


\section{Acknowledgements}

The present work was supported by Grant Agency (No. 1/0281/08 and No. 1/3557/06) of the Ministry of Education, the Research and Development Support Agency (APVV No. 20-038405), Slovak Republic and COST Action D32/011/05 Chemistry in High-Energy Microenvironments.

\section{References}

1. Savage, I.; Thomas, E. J. Asymmetric $\alpha$-Amino acid Synthesis: Synthesis of (+)-Polyoxamic Acid using a [3,3]-Allylic Trifluoroacetimidate Rearrangement. J. Chem. Soc., Chem. Commun. 1989, 717-719.

2. Kakinuma, K.; Koudate, T.; Li, H.-Y.; Eguchi, T. Enantiocontrol by Intrinsic Antiparallel Double Repulsion on Diacetone-D-Glucose Template. Enantioselective Synthesis of Alanine and Chirally Deuterated glycine. Tetrahedron Lett. 1991, 32, 5801-5804.

3. Eguchi, T.; Koudate, T.; Kakinuma, K. The Overman rearrangement on a Diacetone-D-Glucose Template: Kinetic and Theoretical Studies on the Chirality Transcription. Tetrahedron 1993, 49, 4527-4540.

4. Ammenn, J.; Altmann, K.-H.; Belluš, D. Aza-Claisen Rearrangement: Synthesis of 5'-Branched 5'-Aminothymidines. Helv. Chim. Acta 1997, 80, 1589-1606.

5. Chen, A.; Thomas, E. J.; Wilson, P. D. Stereoselective synthesis of thymine polyoxin C using an allylic trifluoroacetimidate-trifluoroacetamide rearrangement. J. Chem. Soc., Perkin Trans 1 1999, 3305-3310.

6. Chida, N.; Takeoka, J.; Tsutsumi, N.; Ogawa, S. Total synthesis of (+)-Lactacystin from DGlucose. J. Chem. Soc., Chem. Commun. 1995, 793-794.

7. Oishi, T.; Ando, K.; Inomiya, K.; Sato, H.; Iida, M.; Chida, N. Total Synthesis of Sphingofungin E from D-Glucose. Org. Lett. 2002, 4, 151-154.

8. Oishi, T.; Ando, K.; Inomiya, K.; Sato, H.; Iida, M.; Chida, N. Total Synthesis of (+)-Myriocin and (-)-Sphingofungin E from Aldohexoses Using Overman Rearrangement as the Key Reaction. Bull. Chem. Soc. Jpn. 2002, 75, 1927-1947.

9. Ohyabu, N.; Nishikawa, T.; Isobe, M. First Asymmetric Total Syntheis of Tetrodotoxin. J. Am. Chem. Soc. 2003, 125, 8798-8805.

10. Overman, L. E. A General Method for the Synthesis of Amines by the Rearrangement of Allylic Trichloroacetimidates. 1,3 Transposition of Alcohol and Amine Functions. J. Am. Chem. Soc. 1976, 98, 2901-2910.

11. Nagashima, H.; Wakamatsu, H.; Ozaki, N.; Ishii, T.; Watanabe, M.; Tajima, T.; Itoh, K. Transition Metal Catalyzed Radical Cyclization: New Preparative Route to $\gamma$-Lactams from Allylic Alcohols via the [3,3]-Sigmatropic Rearrangement of Allylic Trichloroacetimidates and the Subsequent Ruthenium-Catalyzed Cyclization of $\mathrm{N}$-allyltrichloroacetamides. J. Org. Chem. 1992, 57, 1682-1689.

12. Gonda, J.; Helland, A.-Ch.; Ernst, B.; Belluš, D. Diastereoselective Synthesis of 1,2-Diamines by Palladium Catalyzed Aza-Claisen Rearrangement. Synthesis 1993, 729-733. 
13. Anderson, C. E.; Overman, L. E. Catalytic Asymmetric Rearrangement of Allylic Trichloroacetimidates. A Practical Method for Preparing Allylic Amines and Congeners of High Enantiomeric Purity. J. Am. Chem. Soc. 2003, 125, 12412-12413.

14. Jaunzeme, I.; Jirgensons, A. Ether-directed diastereoselectivity in catalysed Overman rearrangement: Comparative studies of metal catalysts. Tetrahedron 2008, 64, 5794-5799.

15. Takano, S.; Akiyama, M.; Ogasawara, K. A New $\alpha$-Amino Acid Synthesis via an Acetimidate Rearrangement. J. Chem. Soc., Chem. Commun. 1984, 770-771.

16. Gonda, J.; Martinková, M.; Zadrošová, A.; Šoteková, M.; Raschmanová, J.; Čonka, P.; Gajdošíková, E.; Kappe, C. O. Microwave accelerated aza-Claisen rearrangements. Tetrahedron Lett. 2007, 48, 6912-6915.

17. Nishikawa, T.; Asai, M.; Ohyabu, M.; Isobe, M. Improved Conditions for Facile Overman Rearrangement. J. Org. Chem. 1998, 63, 188-192.

18. Jacobson, M. A.; Williard, P. G. Synthesis of 2,3-Disubstituted Pyrroles from 3, $N$-Dilithio- $N$ (tert-butyldimethylsilyl)-2-buten-1-amine. J. Org. Chem. 2002, 67, 32-37.

19. Chambers, D. J; Evans, G. R.; Fairbanks, A. J. An approach to the synthesis of $\alpha-(1-6)-C$ disaccharides by tandem Tebbe methylenation and Claisen rearrangement. Tetrahedron 2005, 61, 7184-7192.

20. Villemin, D.; Hachemi, M. Potassium Fluoride on Alumina: One-Pot Synthesis of N-Allyl Trichloroacetamides by Cascade Condensation-Sigmatropic Rearrangement. Synth. Commun. 1996, 26, 1329-1334.

Sample Availability: Samples of compounds 2d, 14a,b are available from the authors.

(C) 2008 by the authors; licensee Molecular Diversity Preservation International, Basel, Switzerland. This article is an open-access article distributed under the terms and conditions of the Creative Commons Attribution license (http://creativecommons.org/licenses/by/3.0/). 